\title{
Dielectronic recombination cross sections of fluorinelike xenon
}

\author{
D. R. DeWitt, D. Schneider, M. H. Chen, and M. B. Schneider \\ Lawrence Livermore National Laboratory, University of California, Livermore, California 94550 \\ D. Church and G. Weinberg \\ Texas A\&M University, College Station, Texas 77843 \\ M. Sakurai \\ National Institute for Fusion Science, Nagoya 464-01, Japan
}

(Received 13 November 1992)

\begin{abstract}
Measurements of relative dielectronic recombination (DR) cross sections for fluorinelike xenon $\left(\mathrm{Xe}^{45+}\right)$ are presented. Recombination takes place in an electron-beam ion trap, where decay rates are obtained as the ions recombine with beam electrons. Experimental data are obtained by counting the number of ions that remain, as a function of time, following the recombination period. The counting is accomplished through the use of an ion-extraction system. An ultralow beam current, $2 \mathrm{~mA}$, was used to obtain an energy resolution of $10.6 \mathrm{eV}$ full width at half maximum. The experimentally measured resonances are compared to convolved theoretical DR resonance strengths. The agreement with theory is excellent.
\end{abstract}

PACS number(s): 34.80.Kw, 34.80.Dp

Dielectronic recombination (DR) is a resonant electron-ion recombination process that affects the ionization balance of plasmas and is of interest in plasma diagnostics [1,2]. Experiments on DR have recently been reported from groups using traps $[3,4]$ and the electron coolers of ion storage rings [5,6], among others. In this paper we present relative dielectronic recombination [7] cross sections for the $L M M$ resonances of fluorinelike xenon. This work is an extension of the results presented in a previous paper [8]. The present work extends those measurements to a system containing an open subshell created by the removal of an $L$-shell electron. As explained below, this system is more complex theoretically. The experimental procedures used for the measurement of these cross sections are also complicated by the openshell configuration.

The ground state of fluorinelike xenon is $1 s^{2} 2 s^{2} 2 p^{5} J=\frac{3}{2}$. Dielectronic recombination onto this system is given schematically as

$$
\begin{aligned}
A\left(1 s^{2} 2 s^{2} 2 p^{5}\right)+e & \rightarrow A^{* *}\left(1 s^{2} 2 s^{2} 2 p^{4} n l n^{\prime} l^{\prime}\right) \\
& \rightarrow A^{*}\left(1 s^{2} 2 s^{2} 2 p^{5} n^{\prime} l^{\prime}\right)+h v
\end{aligned}
$$

or as

$$
\begin{aligned}
A\left(1 s^{2} 2 s^{2} 2 p^{5}\right)+e & \rightarrow A^{* *}\left(1 s^{2} 2 s 2 p^{5} n l n^{\prime} l^{\prime}\right) \\
& \rightarrow A^{*}\left(1 s^{2} 2 s^{2} 2 p^{5} n^{\prime} l^{\prime}\right)+h v .
\end{aligned}
$$

$K$-shell electrons are not excited in these measurements. The intermediate doubly excited state couples as a fourelectron system since two vacancies in the $L$ shell are coupled to two excited electrons. When compared to dielectronic recombination onto a neonlike ion this system involves a much more complex level structure, and decay to a multitude of singly excited states is possible.
735 doubly excited states, from the $2 s^{2} 2 p^{4} 3 l 3 l^{\prime}$ and $2 s 2 p^{5} 3 l 3 l^{\prime}$ configurations, whose theoretical resonance strengths were calculated using the multiconfiguration Dirac-Fock method (MCDF) $[9,10]$, are expected to dominate the $L M M$ dielectronic recombination spectrum. These resonance strengths were obtained for target ions initially in their ground state. Resonance strengths were also calculated for ions initially in the first $\left(1 s^{2} 2 s^{2} 2 p^{5} J=\frac{1}{2}\right)$ and second $\left(1 s^{2} 2 s 2 p^{6} J=\frac{1}{2}\right)$ excited states. These calculations predict that the strongest $L M M$ DR resonances for this ion result from initially excited states. Since a certain fraction of the fluorinelike ions may be expected to be in excited states due to electron-impact excitation, the presence of resonance lines from these excited ions will indicate what fraction, if any, of the fluorinelike ions are in excited states for these experimental conditions.

The Lawrence Livermore National Laboratory electron-beam ion trap (EBIT) $[11,12]$ is used to produce the ions. A $31-\mathrm{mA}$ electron beam is compressed to approximately a $70-\mu \mathrm{m}$ diameter as it accelerates toward the cylindrical drift tubes that form the trap. Ions formed by electron impact are contained within a $2-\mathrm{cm}$ long trapping region formed by a separately biased central drift-tube segment. Isotopically enriched xenon gas is continuously fed into the trap region. The $31-\mathrm{mA}$ electron beam produces a sufficient number of fluorinelike ions in about $800 \mathrm{msec}$. Care must be taken in the formation of the initial distribution of charge states. While it is desirable to produce a high yield of fluorinelike ions, it is important to produce as few oxygenlike ions as possible. Recombination of electrons onto oxygenlike ions produces fluorinelike ions that cannot be distinguished from the original fluorinelike ion population. This complicates the analysis of the interaction rates, adds more variables 
to the experimental fits, and obscures the relative recombination rates. The charge-state distribution in EBIT is produced as the ions are successively ionized by repeated electron impact. The formation of a given distribution at equilibrium is described by a set of coupled differential equations that include, for example, electron-impact ionization, radiative recombination, and charge exchange [13]. Generally, for gases, there are some ions of each charge state with ionization threshold below the electron-beam energy. Thus an envelope of charge states is obtained whose shape is determined by a host of processes. Except for closed-shell configurations, where ionization energies jump dramatically, the ratio of two adjacent charge-state populations is difficult to control. Figure 1 shows the distribution of charge states obtained for the present experiment. The ratio of the fluorinelike to oxygenlike ions is roughly an order of magnitude. This ratio was obtained by selecting an electron-beam energy of approximately $7.9 \mathrm{keV}$; the ionization energies of the fluorinelike and oxygenlike ions are approximately 7.663 and $7.893 \mathrm{keV}$, respectively [14]. For energies lower than the one used, the number of fluorinelike ions produced is lower than desired. Indeed, the roughly 100 ions detected at the peak is the minimum acceptable yield, since this limits the ion reduction to two orders of magnitude at the strongest resonances. Notice the relatively high ratio of neonlike to fluorinelike ions. This ratio cannot be reduced without substantially increasing the ionization energy. The figure also shows the falloff of the ion population with decreasing charge state. Isotopically enriched xenon was used to facilitate a clean separation of the ions during magnetic analysis. The magnet current was fixed at the maximum of the fluorinelike xenon peak for the

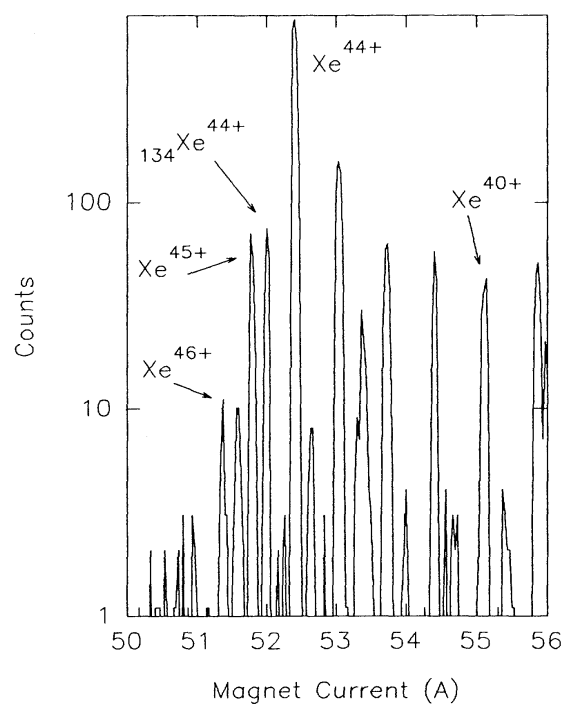

FIG. 1. Xenon charge-state distribution. The distribution peaks at neonlike xenon $\left(\mathrm{Xe}^{44+}\right)$. The yields of fluorinelike and oxygenlike ions are one and two orders of magnitude lower, respectively. Isotopically enriched xenon, with $90 \%{ }_{136} \mathrm{Xe}$ and $10 \%{ }_{134} \mathrm{Xe}$, is used in order to reduce the overlap of the ion peaks. The ions were produced in $800 \mathrm{msec}$, using a $31-\mathrm{mA}$ electron beam at $7.9 \mathrm{keV}$. recombination measurements.

Recombination takes place in the trap, following production of the ions as described above. The selected electron-ion interaction energy was obtained by applying the corresponding voltage to the drift tubes. This energy was scanned to obtain the DR spectrum. In order to improve the energy resolution of the measurements, the electron-beam current was reduced to $2 \mathrm{~mA}$ during the recombination period. This reduced the space charge contribution to the interaction energies. The trapping potential was set to approximately $10 \mathrm{~V}$ to further minimize the range of interaction energies. Most of the remaining energy width of the measured resonances was due to external effects such as power supply noise. Following the recombination period the electron beam was turned off (to prevent further interactions with the remaining ions) and the ions were dumped from the trap [15]. All the ions were then extracted and the fluorinelike ions magnetically separated and counted; this cycle was repeated at each energy. Several scans of this form were obtained. They differed only in the length of time in which recombination was allowed to occur. Scans were obtained for interaction times of $300,340,380$, and 400 msec. From the rate equation that describes the decay of the ion population it is expected that the number of fluorinelike ions remaining in the trap after a time $t$ is given by

$$
n(t)=n_{0} e^{-\varphi t}+n_{b},
$$

where $n$ is the number of fluorinelike $\left(\mathrm{Xe}^{45+}\right)$ ions, $n_{0}$ is the initial number of fluorinelike ions, $\varphi$ is the total decay rate at a given energy, and $n_{b}$ is the number of background or noise counts. The value of $n_{0}$ is expected to be the same, within statistics, at each energy. Its value is found by extrapolating an exponential decay curve to the data at each energy value back to $t=0$ and calculating the average of this value across the energy range. A smooth curve, which varies by about $10 \%$, is found for $n_{0}$ across the energy range in this experiment. This smooth curve, expressed as a third-order polynomial, will be used in the fits of the decay curve, Eq. (2), to the data at each energy. The value of $n_{b}$ was estimated to be 2 counts and the same value was used across the energy range. This is equivalent to subtracting 2 counts from all of the data and fitting a straight line to the logarithm of the data at each energy.

Figure 2 shows the total decay rates. The value of $\varphi$ obtained at energy $\varepsilon$ is equal to the total decay rate at that energy:

$$
\varphi(\varepsilon)=\frac{J^{\prime}}{e}\left[\sigma^{\mathrm{DR}}(\varepsilon)+\sigma^{\mathrm{RR}}(\varepsilon)\right]+\frac{1}{\tau} .
$$

Here $\sigma^{\mathrm{DR}}(\varepsilon)$ is the total DR cross section at energy $\varepsilon$, $\sigma^{\mathrm{RR}}(\varepsilon)$ is the radiative recombination cross section at energy $\varepsilon, \tau$ is the characteristic decay time for other nonresonant processes such as charge exchange and the escape of ions from the trap, $e$ is the electron charge, and $J^{\prime}$ is the effective electron-beam current density. $J^{\prime}$ is not simply the electron-beam current divided by its area since the ions may orbit about the electron beam and are not 


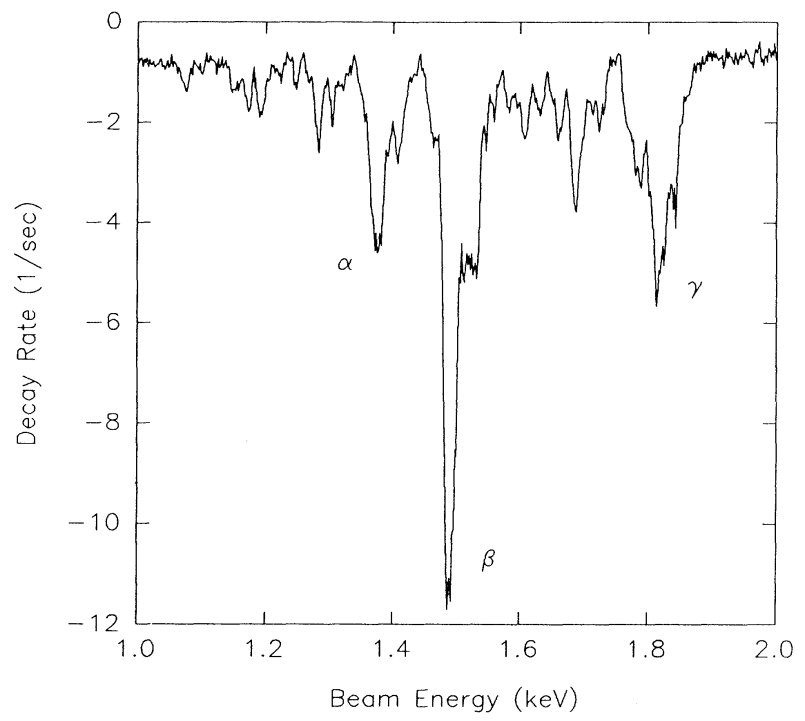

FIG. 2. Total ion decay rate as a function of beam energy. This decay rate, $\varphi(\varepsilon)$, includes decay of ions due to radiative recombination, charge exchange, and leakage of ions from the trap, as well as dielectronic recombination. The major features are identified as $\alpha, \beta$, and $\gamma$ for reference.

necessarily embedded within it. The experimental DR cross section is obtained by solving Eq. (3) for $\sigma^{\mathrm{DR}}(\varepsilon)$ :

$$
{ }^{e} \sigma^{\mathrm{DR}}(\varepsilon)=\frac{e}{J^{\prime}} \varphi(\varepsilon)-\left[\sigma^{\mathrm{RR}}(\varepsilon)+\frac{e}{J^{\prime} \tau}\right] .
$$

The theoretical resonance strengths are computed using autoionization and radiative decay rates. The theoretical DR resonance strength from the initial state $i$ via intermediate state $d$ to the stabilized final state $f$ can be written as a product of dielectronic capture strength multiplied by the fluorescence yield, and is given in atomic units by

$$
S_{i}(d, f)=\frac{\pi^{2}}{E_{i}} \frac{g_{d}}{2 g_{i}} \frac{A_{A}(d \rightarrow i) A_{r}(d \rightarrow f)}{\sum_{k} A_{r}(d \rightarrow k)+\sum_{m} A_{A}(d \rightarrow m)},
$$

where $g_{d}$ and $g_{i}$ are the statistical weight factors, $E_{i}$ is the resonance energy, and $A_{r}$ and $A_{A}$ are the radiative and Auger rates, respectively. In the present work the Auger and radiative rates for each autoionizing state were explicitly calculated from the first-order perturbation theory using the MCDF model $[9,10]$. The energy levels and bound-state wave functions were evaluated in intermediate coupling with configuration interaction from the same complex using the MCDF model in the extended average-level scheme [9]. The Breit interaction and quantum-electrodynamic corrections were included in the calculations of transition energies. The calculated Auger and radiative rates were then employed to compute the DR strength according to Eq. (5). The widths of the theoretical DR cross sections are more than two orders of magnitude smaller than our experimental energy width. The resonance strengths are therefore obtained by integrating over the narrow width of the theoretical cross

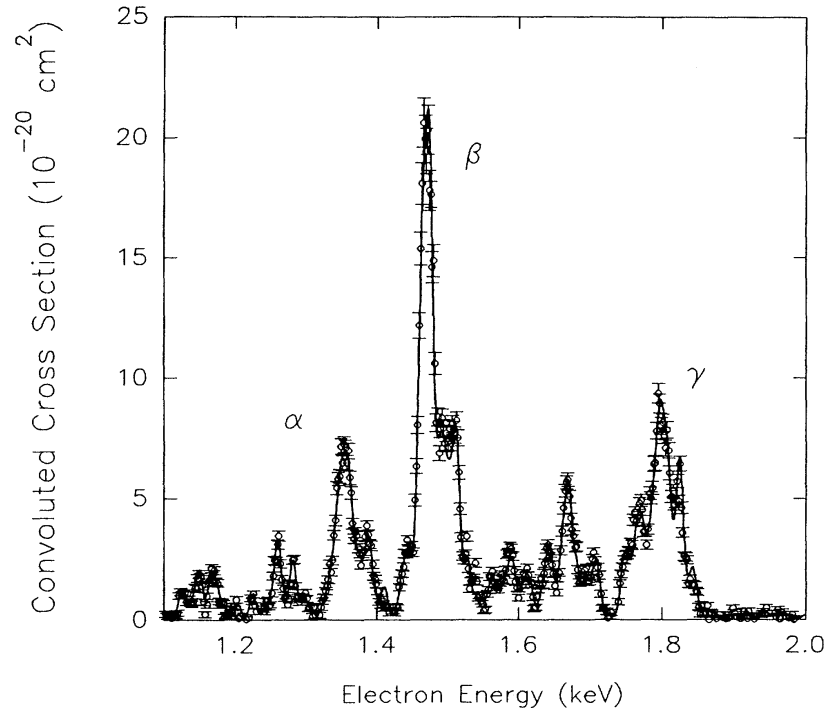

FIG. 3. Fit of the experimental data to theory for the $L M M$ group of resonances. The values of $w$, the experimental energy resolution, and $J^{\prime}$, the effective electron-beam current density, were found using a weighted least-squares fit of the experimental data to the total convolved theoretical DR cross section. The values obtained were $J^{\prime}=8.5 \mathrm{~A} / \mathrm{cm}^{2}$ and $w=4.5 \mathrm{eV}$. The energy resolution is $10.6 \mathrm{eV}$ FWHM.

section. The resulting strengths are convoluted with a Gaussian function for comparison with the experimental data.

In order to compare the data to theory the nonresonant radiative and charge-exchange or leakage decay contributions, represented by the background decay rates in Fig. 2, to the total decay rate, are subtracted. The remaining decay rate is then fitted to the convolved theoretical resonance strengths. Values of $J^{\prime}$, the overall normalization parameter, and $w$, the standard deviation, are determined through the fitting process. The values obtained were $J^{\prime}=8.5 \mathrm{~A} / \mathrm{cm}^{2}$ and $w=4.5 \mathrm{eV}$, which gives an energy resolution of $10.6 \mathrm{eV}$ full width at half maximum (FWHM). This resolution is higher than that reported earlier for our DR measurements on neonlike xenon. This higher resolution results in part from the use of a $60 \%$ lower electron-beam current. The effective electron-beam current density obtained, $J^{\prime}$, is approximately $64 \%$ lower than that obtained in the higher current measurement; the difference is within the expected error. Figure 3 shows the comparison of the normalized experimental data to theory. The amplitudes of the different peaks are in excellent agreement with theory if it is assumed that none of the initial fluorinelike ions are in metastable excited states. This assumption is justified by the fact that the strongest resonance strengths occur for singly excited target ions, making detection certain. If a significant number of fluorinelike ions are in metastable states the total decay rate will be determined by a weighted average of the resonance strengths of the three initial excited-state populations, with the weights given by the relative excited-state populations. The excitation energies of the first and second excited states of the fluorine- 
like ions are calculated to be 0.3349 and $0.6422 \mathrm{keV}$, respectively. Since the DR resonances are in the 1.0-2.0$\mathrm{keV}$ energy range, it may be expected that ions in the ground state are continuously excited by electron impact, inducing an equilibrium distribution of ions in excited states. However, the second excited state may decay to the ground state through an electric dipole transition with a calculated rate of $1.922 \times 10^{12} \mathrm{sec}^{-1}$, while the first excited state may decay to the ground state through a magnetic dipole transition with a calculated rate of $3.467 \times 10^{8} \mathrm{sec}^{-1}$. From the decay rates shown in Fig. 2 it can be estimated that, at an electron energy near that of the strongest cluster of resonances, denoted $\beta$, the average time between electron-ion collisions for a fluorinelike ion is roughly $100 \mathrm{msec}$. The strengths of these resonances are on the order of $1.0 \times 10^{-18} \mathrm{~cm}^{2}$. The lifetime of the metastable first excited state is, on the other hand, less than $10^{-8} \mathrm{sec}$. Therefore, unless the cross section for electronic excitation is many orders of magnitude larger than $10^{-18} \mathrm{~cm}^{2}$, essentially no ions will survive the long interval between excitation and recombination.

In conclusion, we have measured relative dielectronic recombination cross sections in fluorinelike xenon using an electron-beam ion trap. Our energy resolution is 10.6 eV FWHM. Comparison of the measured relative contributions of the $L M M$ resonances with theoretical groundstate cross sections shows excellent agreement. It was thus established that no metastable fluorinelike ions participated in the recombination process.

The technical assistance of E. Magee and D. Nelson in the design and performance of this experiment is gratefully acknowledged. This work was performed under the auspices of the U.S. Department of Energy by the Lawrence Livermore National Laboratory under Contract No. W-7405-ENG-48.
[1] A. Burgess, Astrophys. J. 139, 776 (1964).

[2] J. Dubau, and S. Volonte, Rep. Prog. Phys. 43, 199 (1980).

[3] D. A. Knapp, R. E. Marrs, M. A. Levine, C. L. Bennett, M. H. Chen, J. R. Henderson, M. B. Schneider, and J. H. Scofield, Phys. Rev. Lett. 62, 2104 (1989).

[4] R. Ali, C. P. Bhalla, C. L. Cocke, M. Schulz, and M. Stockli, Phys. Rev. A 44, 223 (1991); R. Ali, C. P. Bhalla, C. L. Cocke, and M. Stockli, Phys. Rev. Lett. 64, 633 (1990).

[5] G. Kilgus, J. Berger, P. Blatt, M. Grieser, D. Habs, B. Hockadel, E. Jaeschke, D. Kramer, R. Neumann, G. Neureither, W. Ott, D. Schwalm, M. Steck, R. Stokstad, E. Szmola, A. Wolf, R. Schuch, A. Muller, and M. Wagner, Phys. Rev. Lett. 64, 737 (1990).

[6] L. H. Andersen, J. Bolko, and P. Kvistgaard, Phys. Rev. A 41, 1293 (1990).

[7] D. R. DeWitt, D. Schneider, M. W. Clark, M. H. Chen, and D. Church, Phys. Rev. A 44, 7185 (1991).

[8] D. R. DeWitt, D. Schneider, M. H. Chen, M. W. Clark, J.
W. McDonald, and M. B. Schneider, Phys. Rev. Lett. 68, 1694 (1992).

[9] M. H. Chen, Phys. Rev. A 31, 1449 (1985); 33, 994 (1986).

[10] I. P. Grant, B. J. McKenzie, P. H. Norrington, D. F. Mayers, and N. C. Pyper, Comput. Phys. Commun. 21, 207 (1980).

[11] R. E. Marrs, M. A. Levine, D. A. Knapp, and J. R. Henderson, Phys. Rev. Lett. 60, 1715 (1988).

[12] M. A. Levine, R. E. Marrs, J. R. Henderson, D. A. Knapp, and M. B. Schneider, Phys. Scr. T22, 157 (1988).

[13] B. M. Penetrante, J. N. Bardsley, D. DeWitt, M. Clark, and D. Schneider, Phys. Rev. A 43, 4861 (1991).

[14] R. D. Cowan, Theory of Atomic Structure and Spectra (University of California Press, Berkeley, CA, 1981).

[15] D. Schneider, D. DeWitt, M. W. Clark, R. Schuch, C. L. Cocke, R. Schmieder, K. J. Reed, M. H. Chen, R. E. Marrs, M. Levine, and R. Fortner, Phys. Rev. A 42, 3889 (1990). 